\title{
NUMERICAL MODELING OF NON-COHESIVE CONTACT IN MULTI-BODY HYDRODYNAMIC SYSTEMS WITH SPH
}

\author{
Mohammad Javad Mohajeri', Mehdi Shafieefar ${ }^{2}$ and Soheil Radfar ${ }^{3}$
}

\begin{abstract}
Enforcing solid boundary conditions is one of the most challenging parts of the Smoothed Particle Hydrodynamics (SPH) method and many different approaches have been recently developed. Better understanding of interaction forces between solid bodies is of great importance in the investigation of structural stability and armor layer displacement in breakwaters. In this study, performance of repulsive force and dynamic boundary conditions have been investigated and showed that non-physical results are presented in non-cohesive contact. In this paper, a noncohesive contact model in multi-body hydrodynamic systems has been developed and validated against other common boundary conditions. Using the developed contact model, the effect of regular and irregular placement of cubic concrete armors has been investigated. Also, comparison has been made with Van Buchem (2009) experimental results and concluded that in the irregular case it is more possible that a unit moves toward instability.
\end{abstract}

Keywords: non-cohesive contact; multi-body systems; rubble-mound breakwater; cube armor units; SPH

\section{INTRODUCTION}

Investigation of structural stability and armor layer displacement in breakwaters, due to wave force effect is of great importance in the design of this type of marine structures. Better understanding of wave's hydrodynamic behavior and interaction forces between solid bodies is a fundamental step in this regard. Accurate boundary conditions are essential since in many applications precise loading on solids is required such as forces on floating bodies or shorelines structures. Many researchers have focused on applying a rigid body boundary condition, which has satisfactory performance, in the SPH method. Monaghan, introduced repulsive force boundary enforcement for modeling of rigid boundary in fluid media (J. J. Monaghan, 1994). Also, in the same method, this force has been applied using a function in the form of the delta function, near boundary particles (Peskin, 1977). Later, Monaghan and Kos modified the interpolation procedure and considered the effect of distance between boundary particles in the wall-induced repulsive force (J. Monaghan \& Kos, 1999). Rogers et al. modified the repulsive force function and calculated the vertical force acting on a water particle (Rogers, Dalrymple, \& Stansby, 2009). Adams and Nosonovsky in their study, presented a comprehensive review on the contact force modeling using the same concept (Adams \& Nosonovsky, 2000). One of the earliest well known theories in this context is Hertz contact theory (Hertz, 1881).

Most practical and yet the simplest algorithm of dynamic boundary condition have been introduced by Gomez-Gesteira et al. (Gomez-Gesteira et al., 2012). However, in this algorithm, particle penetration problem has not been solved completely. The main advantages of this algorithm are its low computational cost and simple implementation as a computational code, which provide its applications for different rigid boundaries (including gate, wave maker, wall, floating body). Liu and Shao in their study, used a combination of two different types of ghost particles for modeling rigid body boundaries (Liu, Shao, Li, \& Fu, 2011). They concluded that with this boundary enforcement model, pressure variations are reduced in the vicinity of rigid boundary compared with repulsive force boundary enforcement algorithm. Generally, using concepts like ghost particles, is very complicated and in the cases with sharp edges and/or curved surfaces cause significant error in the calculation of the acting force on the rigid body.

Hashemi et al., using a boundary condition based on lubrication force, simulated motion of rigid bodies in incompressible non-Newtonian fluid flows (Hashemi et al., 2012). In this method, similar to the repulsive force method, a layer of SPH particles were defined as rigid boundary.

Pazouki et al. proposed a fully Lagrangian method to solve fluid-solid interaction problems. This SPH-based method provides the capability of modeling the interaction of rigid and elastic bodies in the framework of a multi body dynamic system (Pazouki, et al., 2014). In this study, based on vertical lubrication force function, proposed by Ladd and Verberg, a function for smooth contact modeling in

\footnotetext{
${ }^{1}$ Department of Marine Structures, Faculty of Civil and Environmental Engineering, Tarbiat Modares University, Tehran, Iran; j.mohajeri@modares.ac.ir

2 Department of Marine Structures, Faculty of Civil and Environmental Engineering, Tarbiat Modares University, Tehran, Iran; shafiee@modares.ac.ir

${ }^{3}$ Department of Marine Structures, Faculty of Civil and Environmental Engineering, Tarbiat Modares University, Tehran, Iran; soheil.radfar@modares.ac.ir
} 
the SPH has been introduced (Ladd \& Verberg, 2001). In the lubrication force function, the vertical force between two approaching spheres increases rapidly and prevents physical impact between two rigid bodies.

Generally, treatments for enforcing solid boundary conditions could be divided into two main categories, based on force calculation algorithm: I) Boundary conditions that calculate the active force between fluid particle and boundary particle using a mathematical equation, e.g. Repulsive Force and Lubrication Force. II) In dynamic boundary conditions, boundary particles are forced to satisfy same equations as fluid particles, e.g. Ghost Particles technique. Pressure fields obtained using dynamic boundary conditions are usually smooth with less numerical fluctuations. However, applying such boundary conditions to manipulate solid-solid interactions is not an appropriate treatment. In this study, the cause of imperfection of such boundary treatments and its results are evaluated by modeling some test cases. Then, a non-cohesive contact model is developed and its performance is compared against available boundary treatment technics. In the last section, application of developed boundary condition is evaluated in the modeling of armor layer of a breakwater.

\section{BOUNDARY CONDITIONS}

One of the most important issues in the SPH method is how to apply the boundary conditions for computational domain and rigid boundaries (fixed and moving). There are two major problems in applying appropriate boundary condition in an SPH-based computational model:

1) The first issue is discontinuity in the vicinity of a particle and thus, error in the approximation function for this point. This problem happens in free surface and rigid boundaries, since there are no particles near these surfaces. To solve this issue in current study, mathematical correction of kernel function has been used.

2) The other issue is to prevent fluid particles penetrating into the rigid boundaries. In different rigid boundary treatment of SPH method, various approaches have been used to prevent fluid particles penetrating into the rigid boundaries. Two most common rigid boundary treatments of SPH method are introduced in Table (1):

\begin{tabular}{|c|c|}
\hline \multicolumn{2}{|c|}{ Table 1. Different common boundary conditions in SPH method } \\
\hline Boundary enforcement & Main feature \\
\hline Repulsive force & Applying the force between particles based on a mathematical relationship \\
\hline Dynamic & Contribution of boundary particles in the solution of fluid equations \\
\hline
\end{tabular}

In dynamic boundary condition, boundary particles are included in the solution of fluid equations. Thus, boundary particles in addition to satisfying momentum and continuity equations, are also included in the equation of state. However, the motion of these particles does not comply fluid motion and move based on fixed boundary particle type (fixed boundaries as domain boundary) and/or as an applied function (e.g. Floating body, wave maker, gate, etc.). The force between boundary particles of a rigid body and fluid particles, like other common methods of computational fluid mechanics is calculated based on the pressure of fluid particles in the vicinity of the rigid boundary particle.

Repulsive force boundary condition has been proposed to ensure that water particles do not penetrate into the rigid boundary. The main difference of these two boundary condition is that in dynamic boundary condition, forces on the rigid body is calculated based on fluid pressure. But in the other one, such calculation is based on a mathematical function, which is dependent on distance and relative velocity of particles.

\section{PERFORMACE OF RIGID BODY BOUNDARY CONDITIONS}

Accuracy and convergence in the dynamic and repulsive force boundary conditions are evaluated via comparison of some test cases, whose experimental and analytical results are available. Velocity field of fluid, fluid force on rigid bodies and interaction of rigid boundaries have been investigated in these cases.

\section{TEST CASE 1: FRICTION BETWEEN TWO SOLID SURFACES}

The physical characteristics of a cylinder, which is located on a slope with rigid boundary, is shown in Figure (1). The rigid body moves from the height of $55 \mathrm{~cm}$, due to its weight and after $0.5 \mathrm{~s}$ reaches $22 \mathrm{~cm}$ from the base level. The scope of the modeling is to investigate the accuracy of this method in calculating vertical force and friction between two rigid surfaces in rotation condition. In the 
algorithm of repulsive force boundary condition, the friction force between the rigid boundaries is estimated based on Columb law and the amount of vertical force calculated in repulsive force function. Therefore, error in calculation of acting force between rigid boundaries, has direct influence on the frictional movement of the body.

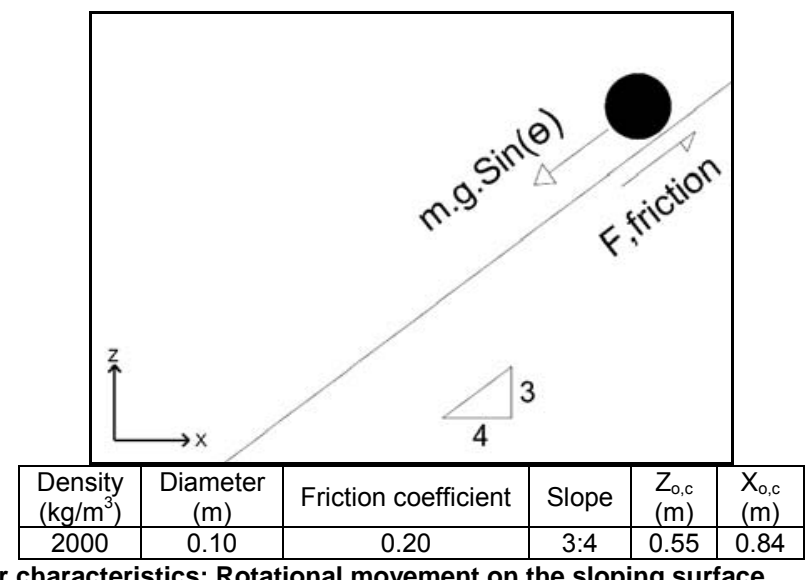

Figure 1. Cylinder characteristics; Rotational movement on the sloping surface

Like previous simulations, performances of repulsive force and dynamic boundary conditions have been investigated and the results for z-coordinate of mass center of body over time, are presented in figure (2). In repulsive force boundary condition, the results are completely compatible with analytical solution for equations of motion of the body. However, in dynamic boundary condition, motion of the body is not predicted correctly and significant error is observed in the results. This error may be originated by the fact that in dynamic boundary condition, physical variables of boundary particles are calculated from the solution of fluid equations. In other words, calculation of forces between two solid boundaries, is solely a function of particle pressures. This fact has only rational meaning for estimation of the fluid forces on a solid body.

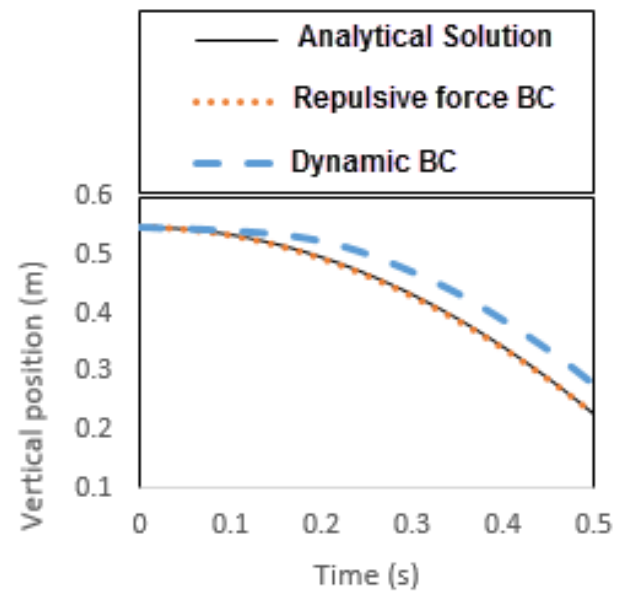

Figure 2. Time history for vertical position of center of mass; rotation on sloping surface

In order to better investigation of friction force calculation, sliding motion due to weight force of a rigid body on a sloping surface has been investigated. Physical characteristics are presented in figure (3). The results for the z-coordinate of mass center in this case is shown in figure (4). As it is expected, again the dynamic boundary condition has a significant error in the estimation of the friction force between two rigid boundaries. As it is seen here, the error in the calculation of the repulsive force boundary condition is rather more than the results of the rotational motion condition. It is due to the error, in mathematical function for calculation of the repulsive force, where the computational error is increased due to increase of the number of particles in contact. 


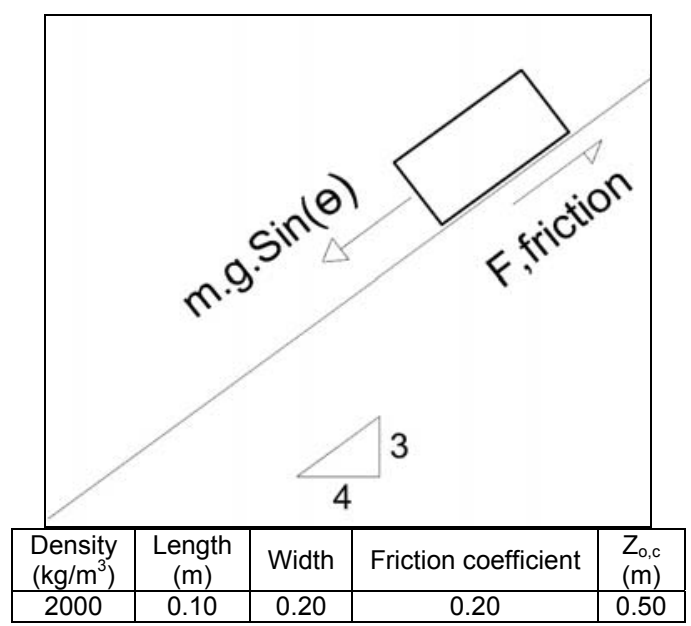

Figure 3. Physical characteristics of the problem; sliding motion on the sloping foreshore

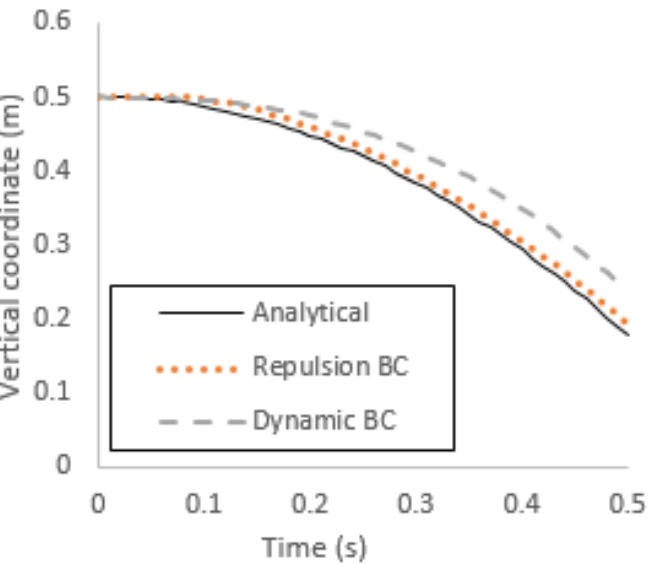

Figure 4. Time history for vertical position of the sliding body on the sloping surface

\section{TEST CASE 2: ROLLING WITH THE INITIAL VELOCITY}

In this test case, in order to estimate the accuracy of the boundary condition in the modeling, estimation of the active forces in contact phenomenon and contact cut off between two parallel rigid bodies, are investigated for a circular body, which turns to suspension state from rotation state. In figure (5), physical characteristics of the problem is presented.

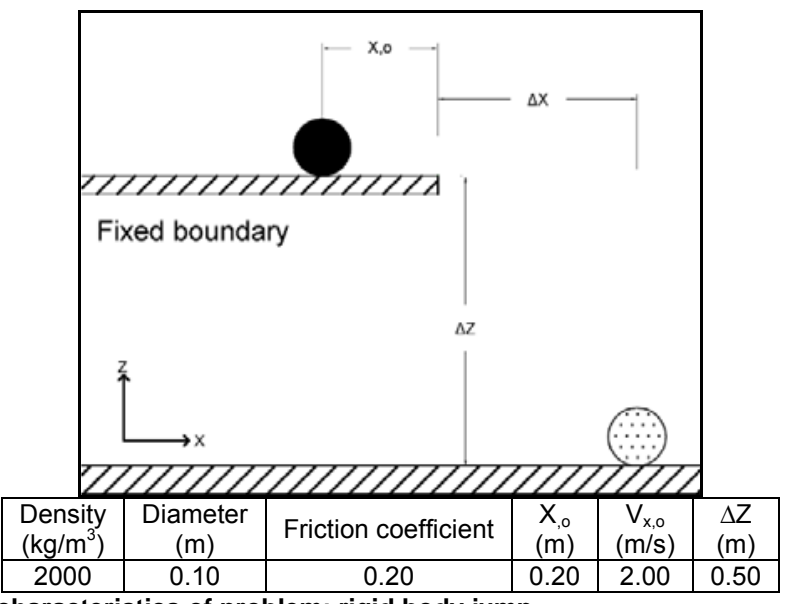

Figure 5. Physical characteristics of problem; rigid body jump 
The body begins its motion with initial velocity of $2 \mathrm{~m} / \mathrm{s}$ and $20 \mathrm{~cm}$ from the edge of fixed boundary. The velocity of the body decreases due to friction force and when it loses its contact with fixed boundary, collides the earth after $0.43 \mathrm{~s}$, at $86 \mathrm{~cm}$ from its initial position. The body motion during its contact with fixed boundary, is calculated from the following equation:

$$
\begin{gathered}
\mathrm{a}_{\mathrm{x}}=-\mu \mathrm{g} \\
\Delta \mathrm{x}=0.5 \mathrm{a}_{\mathrm{x}} \mathrm{t}^{2}+\mathrm{V}_{0} \mathrm{t}
\end{gathered}
$$

Time history diagram for the z-coordinate of mass center and also, diagram of the z-coordinates of mass center vs its x-coordinates, are shown in figures (6) and (7), respectively. As it is obvious, in dynamic boundary condition, non-physical phenomenon of body jump is observed in the beginning of the motion. Despite, appropriate estimation of body collision time in repulsive force boundary method, yet in the calculation of horizontal displacement of the body, there is $10 \mathrm{~cm}$ error, approximately.

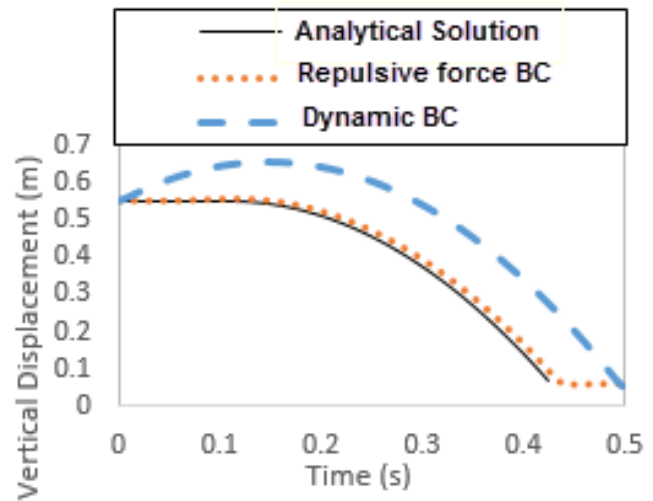

Figure 6. Time history for vertical displacement of center of mass; body jump modeling from rigid boundary

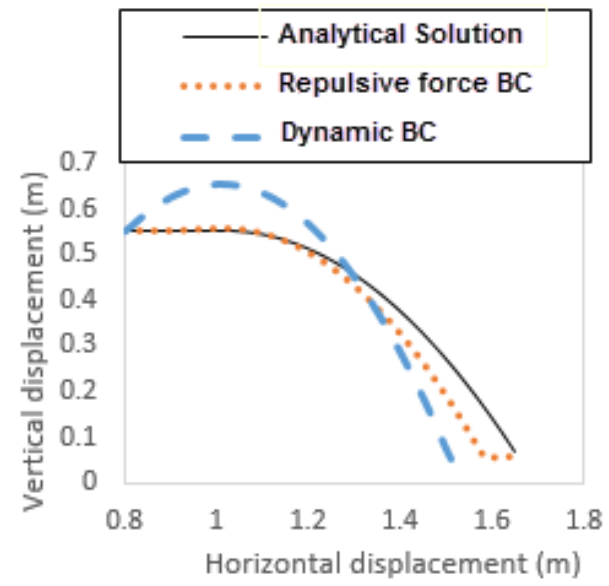

Figure 7. Rigid body motion; body jump modeling from rigid boundary

TEST CASE 3: LOOSING CONTACT IN THE MOTION PERPENDICULAR ON CONTACT SURFACE

In the previous problems, dynamic interaction between rigid bodies, which have vertical motions related to each other and also are in each other's support domain, has been investigate. For example, two similar circular bodies with the diameter of $3 \mathrm{~cm}$ are located on a fixed boundary. Here, minimum distance between the bodies is $1 \mathrm{~cm}$ and the span of kernel function is $13 \mathrm{~mm}$. So, in the calculation of repulsive force function, the two bodies effect on each other. In the first time step, the right body start its motion rightward with the velocity of $2 \mathrm{~m} / \mathrm{s}$. Other physical characteristics of this problem are presented in figure 8 . 


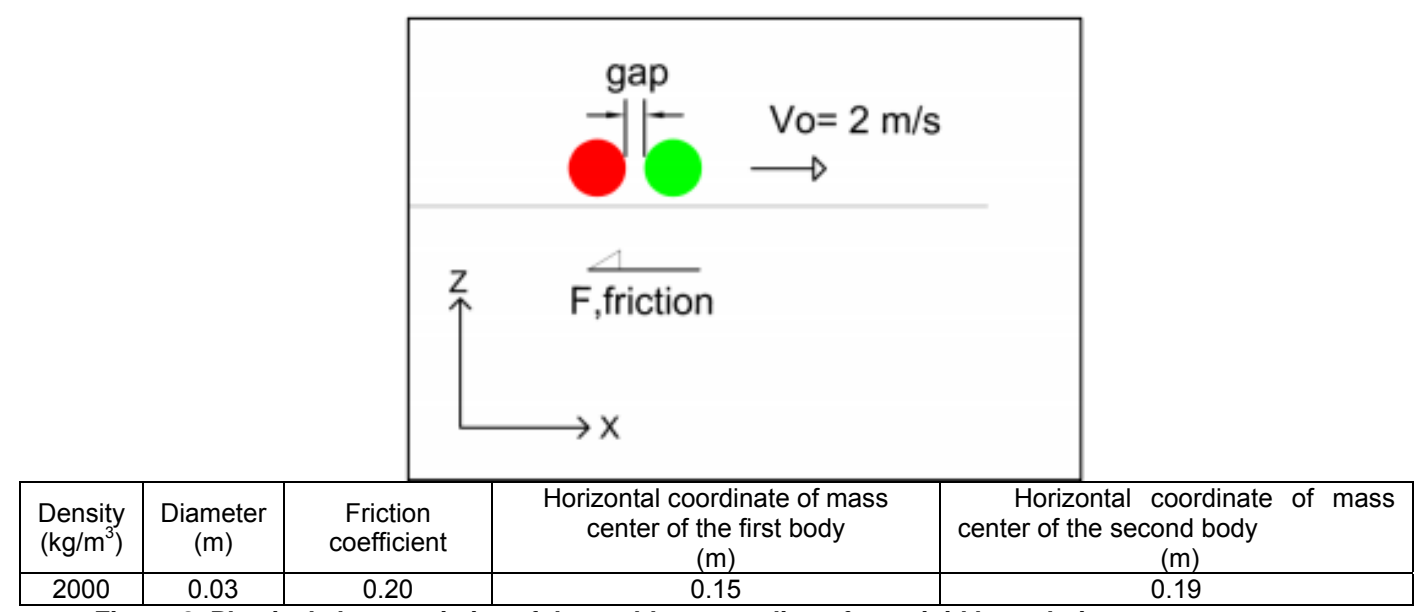

Figure 8. Physical characteristics of the problem; receding of two rigid boundaries

Based on the principals of solid body dynamics, the first body should stay fixed in its position and the second body displaces nearly $75 \mathrm{~cm}$ in $0.5 \mathrm{~s}$. In figure 9, horizontal displacement of the mass center of two cylinders is presented over time. As it is seen, the first body displaces $55 \mathrm{~mm}$ and the second one, displaces $84 \mathrm{~mm}$; this is non-physical phenomenon. This fact leads us to develop a non-cohesive contact model to avoid illogical results.

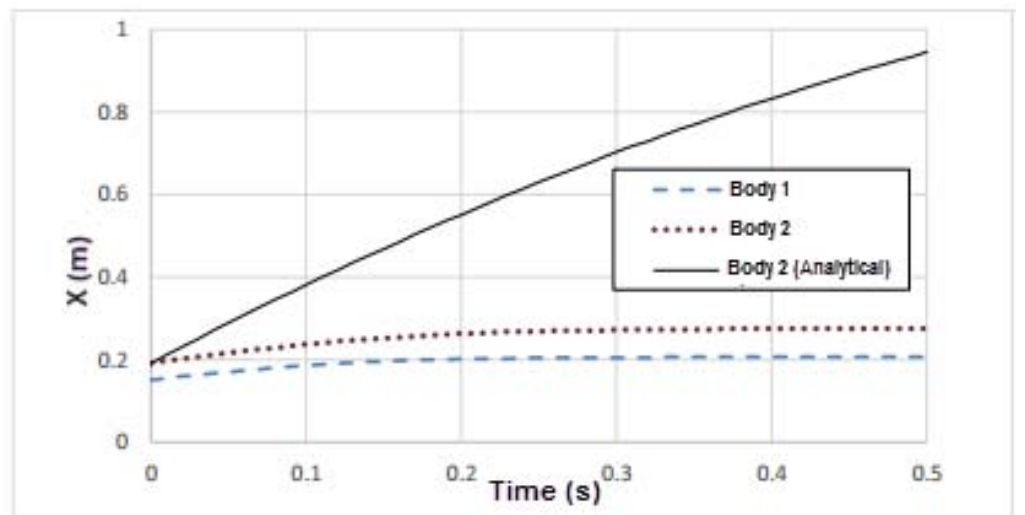

Figure 9. Effect of sharing in kernel region on body motions in the repulsive force boundary condition

\section{DEVELOPING NON-COHESIVE CONTACT MODEL}

As it is discussed in previous section, to avoid non-physical results non-cohesive contact model is required. Available algorithms have some imperfections in modeling non-cohesive contact of rigid bodies. These imperfections are related to using absorption or repulsive force based on the magnitude and sign of the relative normal velocity between two boundary particles. Therefore, a suitable contact algorithm is developed to model interaction of solid rigid bodies in a multi-body hydrodynamic system. In this contact algorithm, normal force between two solid boundary particles is calculated as follow:

$$
\vec{f}=\vec{n} R(\psi) P(\xi) \varepsilon\left(z, u_{L}\right)
$$

Where $\mathrm{n}$ is the normal of the solid wall. The distance $\psi$ is the perpendicular distance of the particle from the wall. $\mathrm{P}$ and $\varepsilon$ functions are corrections for tangential distance $(\Psi)$, relative normal velocity $\left(u_{L}\right)$ and depth of the water $(z)$. The repulsion function, $R(\Psi)$ is evaluated in terms of the normalized distance of two solid boundary particles, $q=\Psi / 2 \mathrm{~h}$.

$$
R(\psi)=A \frac{1}{\sqrt{q}}(1-q)
$$


There are two types of " $\mathrm{A}$ " coefficients: " $\mathrm{A}_{\mathrm{BP}}$ ", coefficient of moving rigid boundary particle, and " $\mathrm{A}_{\mathrm{WP}}$ " coefficient of fluid particle. The focus of this paper is on modifying " $\mathrm{A}_{\mathrm{BP}}$ ", which is formulated as below:

$$
A_{B P}= \begin{cases}(10 / B)^{2}\left(0.01 c_{\text {diff }}-c_{i} u_{L}\right) / h & u_{L}>0 \\ -(10 / B)^{2} r_{f} c_{i} u_{L} / h & u_{L} \leq 0\end{cases}
$$

Here, $h, c_{i}$ and $B$ are smoothing length, sound velocity in fluid and sound coefficient, respectively.

In Eq. (3), when the relative vertical velocity between two bodies $\left(\mathrm{u}_{\mathrm{L}}\right)$ is sufficiently greater than zero, the second term will be more than the first term $\left(0.01 \mathrm{c}_{\text {diff }}\right)$ and thus, "A" will have negative sign. So, the calculated force between two bodies will have negative value, which causes a type of cohesion between two rigid bodies. Therefore, the calculation function of repulsive force in the rigid body boundary condition to remove cohesion between bodies, has to be modified. Here, it is concluded that " $A$ " should be zero when rigid boundaries are about to receding from each other. So, the formulation of " $\mathrm{A}$ " in the repulsive force boundary condition can be rewritten as:

$$
A_{B P}= \begin{cases}0 & , u_{L}>0 \\ (10 / B)^{2}\left(-r_{f} c_{i} u_{L}\right) / h & , u_{L} \leq 0\end{cases}
$$

To investigate effect of modifying the contact model on the simulation of non-cohesive contact, results of the third test case (figure (8)) have been compared for dynamic, repulsive force and modified boundary conditions. As it is seen, results of modified boundary condition has good agreement with analytical solution. While, in the other boundary conditions, significant divergence has been seen from the analytical results (see figure (10)).

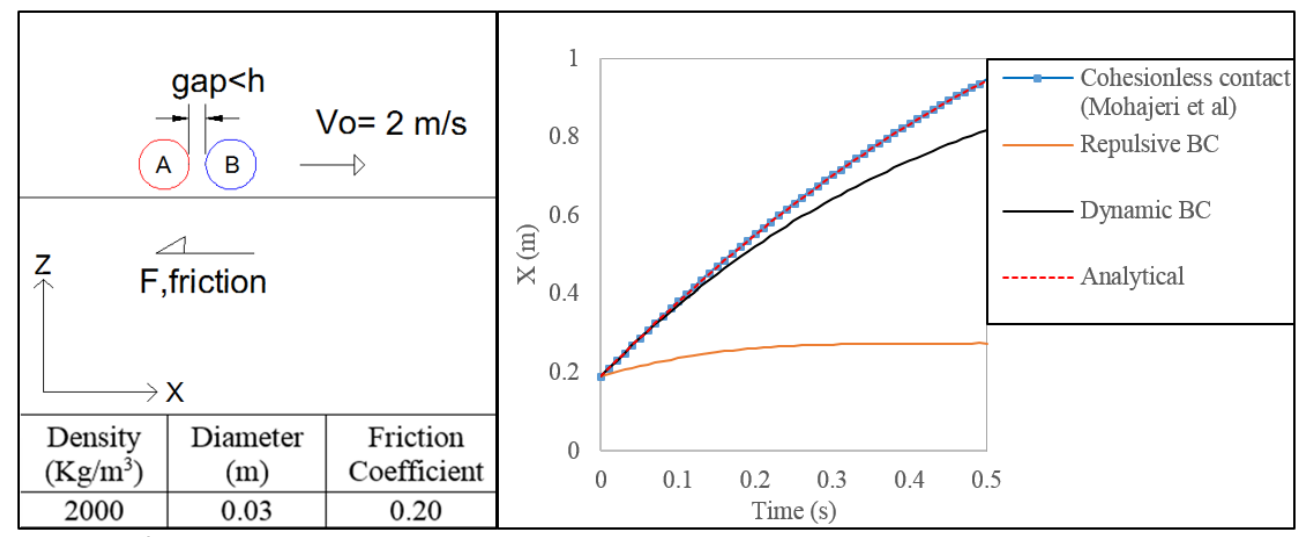

Figure 10. Contact in a dynamic system: Left: physical characteristics of simulation, Right: Time history of $\mathrm{X}$-coordinate of the center of disk "B"

Although, modified boundary condition (Eq. (5)) has the capability of modeling the vertical motion of non-cohesive rigid bodies in a dynamic system, however, Repulsive and Dynamic boundary conditions are less efficient in modeling multi-body systems in static (or hydrostatic) condition. Further investigations showed that in this case, rigid boundary particles penetrate in each other. Overcoming this limitation, further modifications have been made in coefficient "A". Eq. (6) shows the new formulation of "A". In this modified formulation, the boundary force function among rigid bodies, do not behave as a repulsive force, anymore and it is more the same as a compressive spring model (see figure 11).

$$
A_{B P}=\left\{\begin{array}{l}
0 \quad, u_{L}>0 \\
(10 / B)^{2}\left(0.005 c_{0}^{2}-r_{f} c_{i} u_{L}\right) / h \quad, u_{L} \leq 0
\end{array}\right.
$$

Here, the added term is an imaginary static term and coefficient " $A$ " act as a smooth spring, which is activated only in pressure. Selecting bigger values for "A", boundary force function acts as a high stiffness spring. This causes impact mode in body contacts and floating point overflow in the 
calculation. If the selected value for "A" is small, penetration occurs due to reduction in spring stiffness.

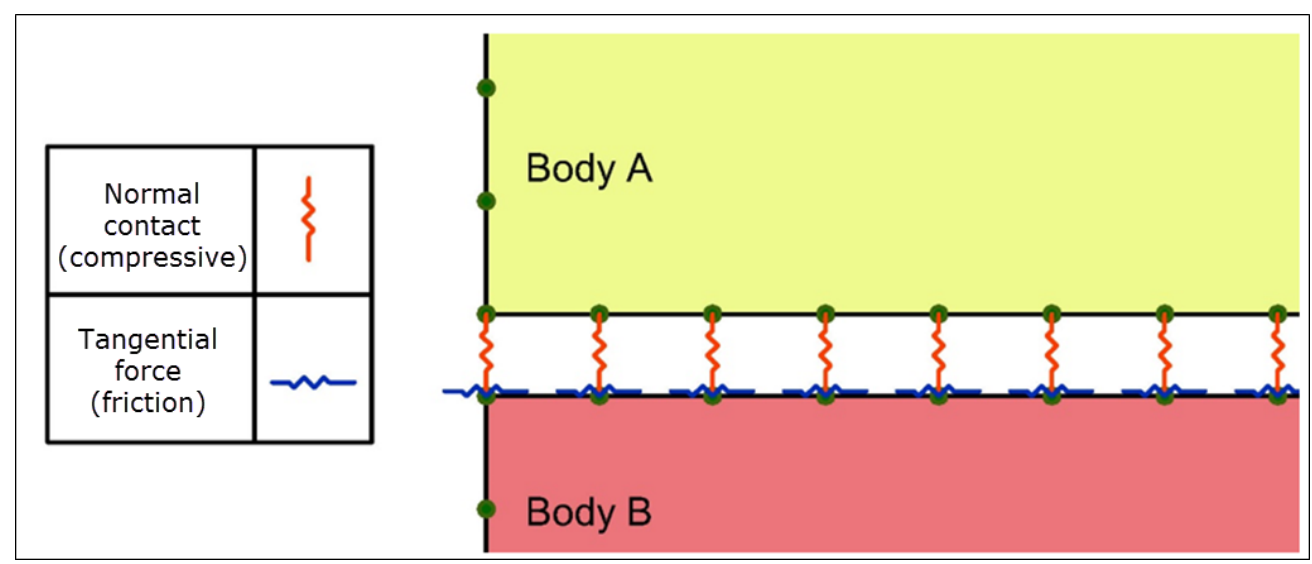

Figure 11. Schematic view of the developed contact model

For validation purposes, the accuracy of the developed contact algorithm is evaluated in simulating rigid bodies' interaction in a hydrostatic condition. The physical characteristics of this test case are shown in figure 12. The proportion of the active vertical body forces on the weight of the body in the numerical modeling has maximum of 0.0015 which is a negligible error (figure 13). The velocity field of this model at $\mathrm{t}=0.30 \mathrm{sec}$ is shown in figure 14 .

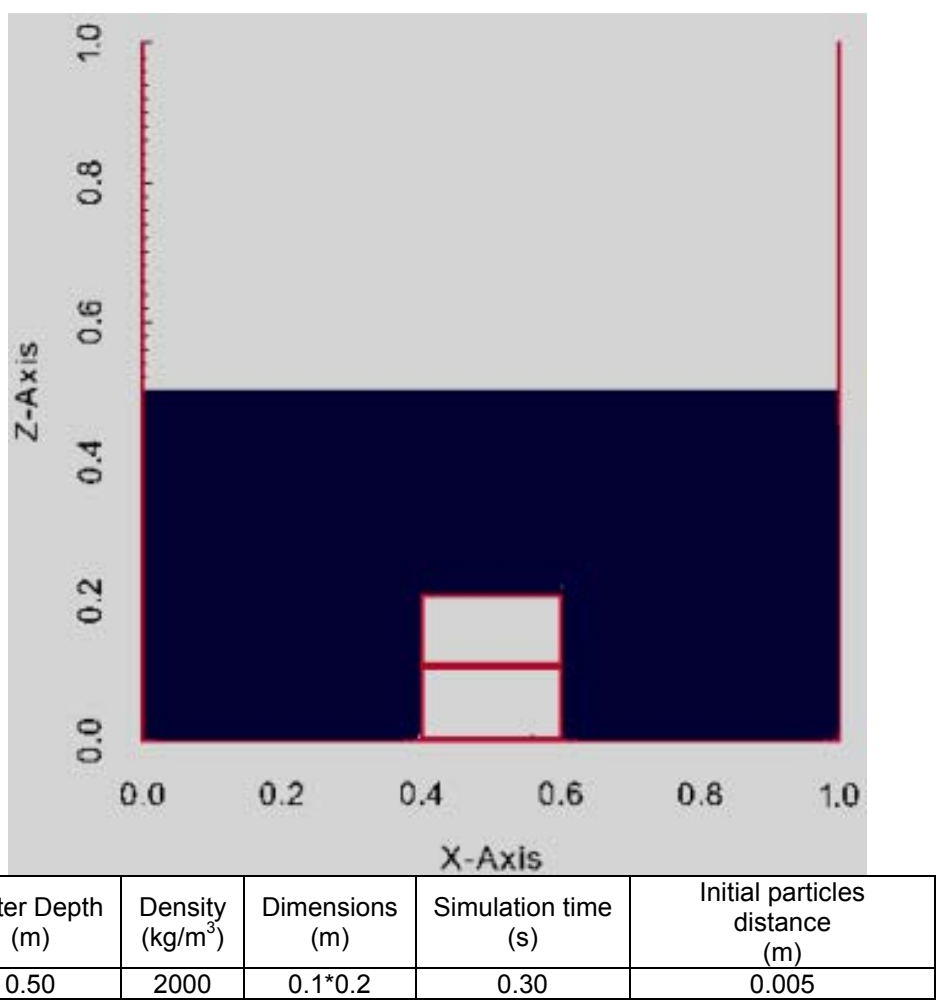

Figure 12. Physical characteristics; rigid body jump 


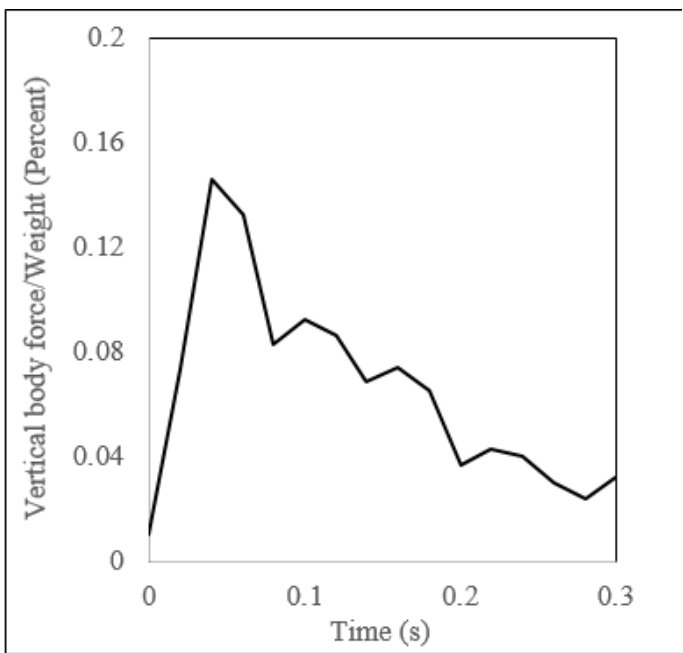

Figure 13. Accuracy of the developed contact algorithm is evaluated in simulating rigid bodies' interaction in a hydrostatic condition

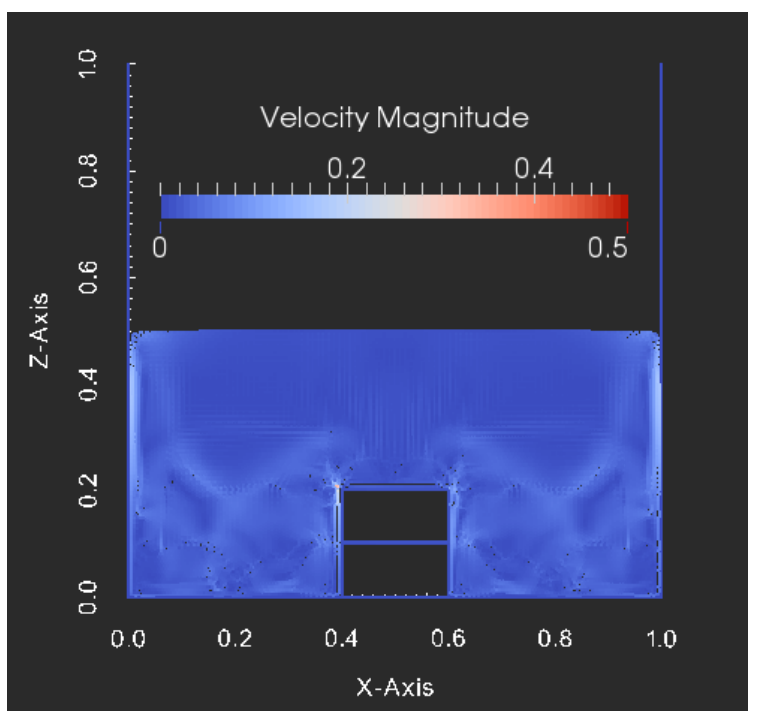

Figure 14. Contact in a hydrostatic system: Velocity field $(\mathrm{m} / \mathrm{s})$ at $\mathrm{t}=0.30 \mathrm{sec}$

\section{ENGINEERING APPLICATION: ARMOR STABILITY IN RUBBLE MOUND BREAKWATERS}

In this section, using the developed contact model, effect of armor layout on displacement of cubic concrete armors has been investigated. Physical characteristics of the simulated breakwater are based on Van Buchem study (Van Buchem, 2009). Initial particle characteristics and these physical characteristics are shown in figure 15.

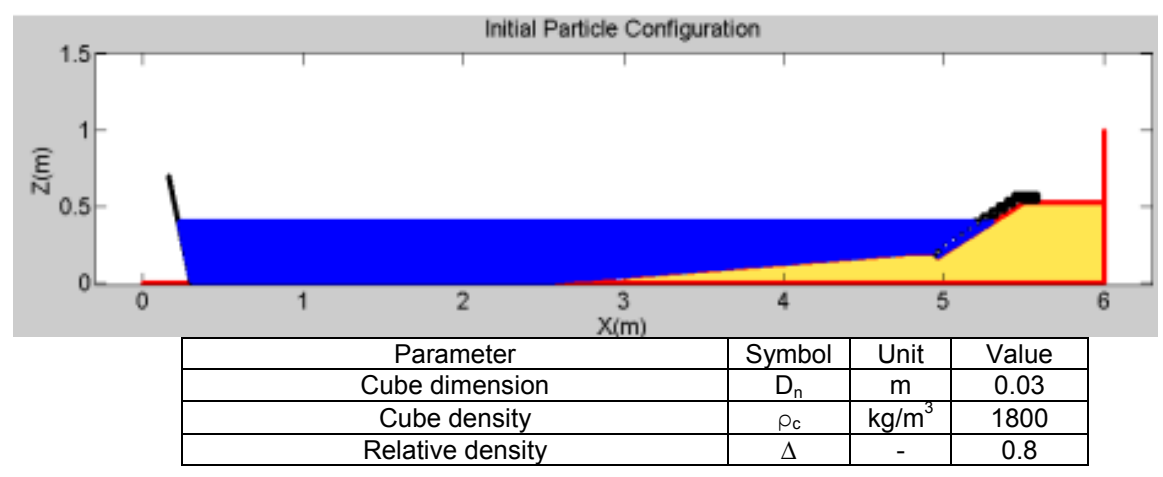




\begin{tabular}{|c|c|c|c|}
\hline Water density & $\rho_{\mathrm{w}}$ & $\mathrm{kg} / \mathrm{m}^{3}$ & 1000 \\
\hline Water level at tow & $\mathrm{h}_{\mathrm{c}}$ & $\mathrm{m}$ & 0.2 \\
\hline Water depth at wave maker & $\mathrm{h}_{\mathrm{o}}$ & $\mathrm{m}$ & 0.6 \\
\hline Beach slope & $\mathrm{a}_{\mathrm{o}}$ & $\mathrm{deg}$ & 5 and 10 \\
\hline Breakwater Slope & $\mathrm{\alpha}$ & - & $1: 1.5$ \\
\hline Distance of free surface from superstructure & $\mathrm{R}_{\mathrm{c}}$ & $\mathrm{m}$ & 0.13 \\
\hline
\end{tabular}

Figure 15. Physical characteristics of the breakwater

In the first simulation, irregular placement of armor units is as figure 16 . To ensure the stability of armor units in hydrostatic condition, a simulation without wave presence has been conducted. Position of rigid boundary particles are shown in figures 17 and 18 for two time steps. Porosity of armor layer $\left(\mathrm{n}_{\mathrm{p}}\right)$ is considered 0.25 . Neglecting few displacements of armor units in the beginning of simulation $(\mathrm{t}=0.5 \mathrm{~s})$, no significant displacements have been seen in the armor layer, in the rest of simulation.

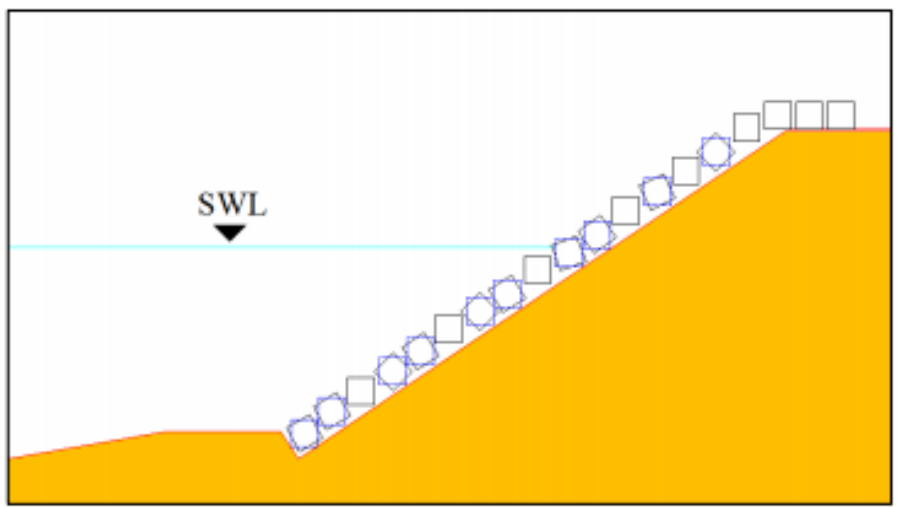

Figure 16. Irregular placement of armor units in the beginning of simulation

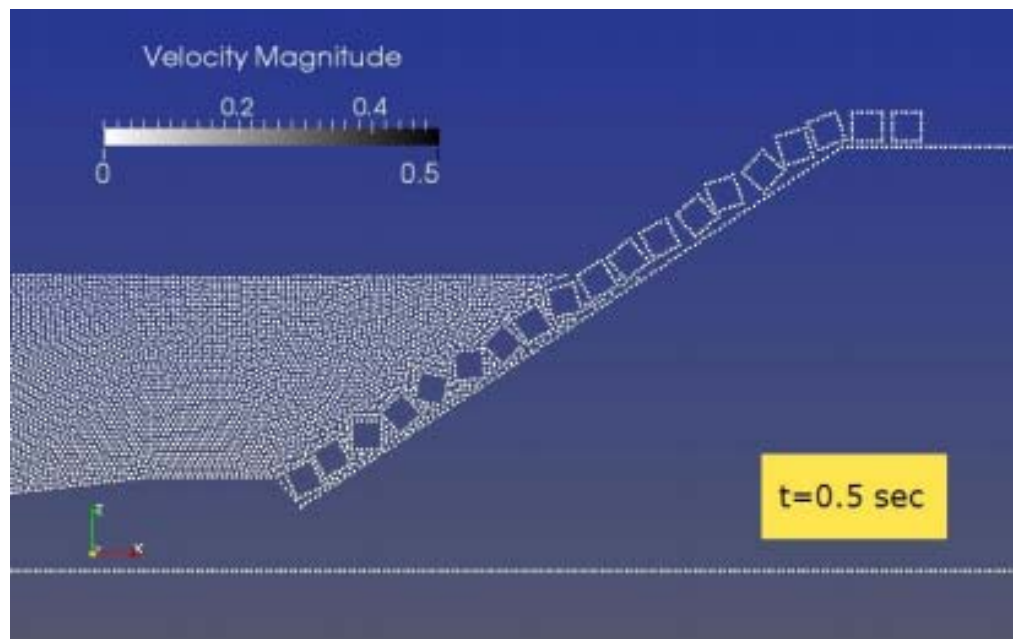

Figure 17. Investigation of structural stability of armor layer in irregular placement $(t=0.5 s)$ 


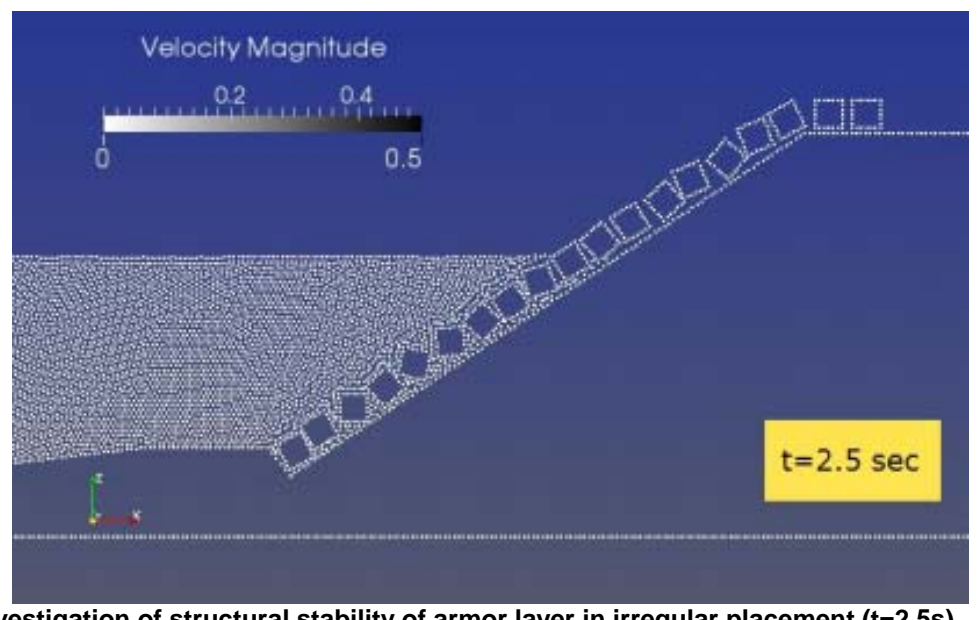

Figure 18. Investigation of structural stability of armor layer in irregular placement $(t=2.5 s)$

In the second case, a regular placement is considered (see figure 19). After nearly $0.5 \mathrm{~s}$ of simulation, armor layer has been stable with a few deformation. Porosity of armor layer $\left(\mathrm{n}_{\mathrm{p}}\right)$ in this case is a bit less than the first case and is 0.21 . Velocity field and magnitude of boundary particles are presented in figure 20. As it is seen, boundary velocity of rigid bodies and also fluid particles, near the armor layer, is very close to zero. This fact shows that the hydrostatic condition has been satisfied in this region.

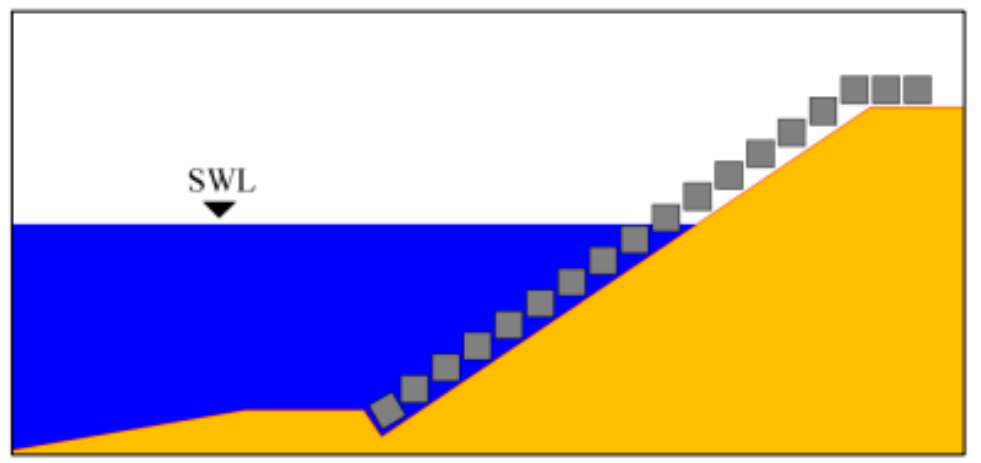

Figure 19. Regular placement of armor layer

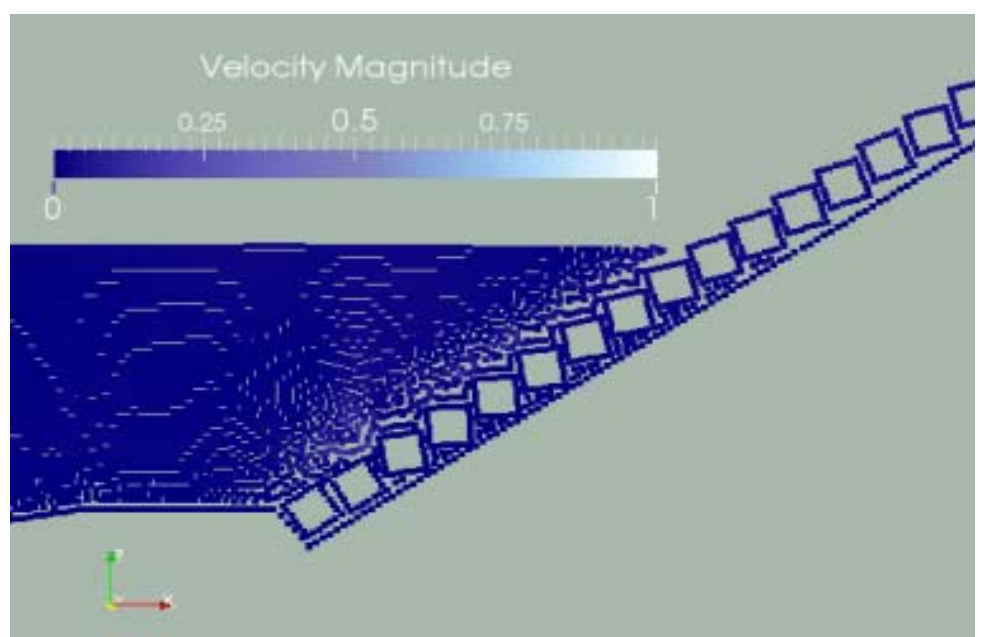

Figure 20. Velocity magnitude in stable condition of armor layer with regular placement $(t=0.52 s)$

Displacement of armor layer in different time step for both regular and irregular placements are shown in figures 21. As it is observed, one of the armor units in irregular case is excluded from its 
original location and caused overall instability in the armor layer. Owing to this fact that the wave height at the tow of breakwater is the criterion for stability calculation in the armor layer, stability parameter in these models is equal to 5 (for a wave height of $12 \mathrm{~cm}$ ).
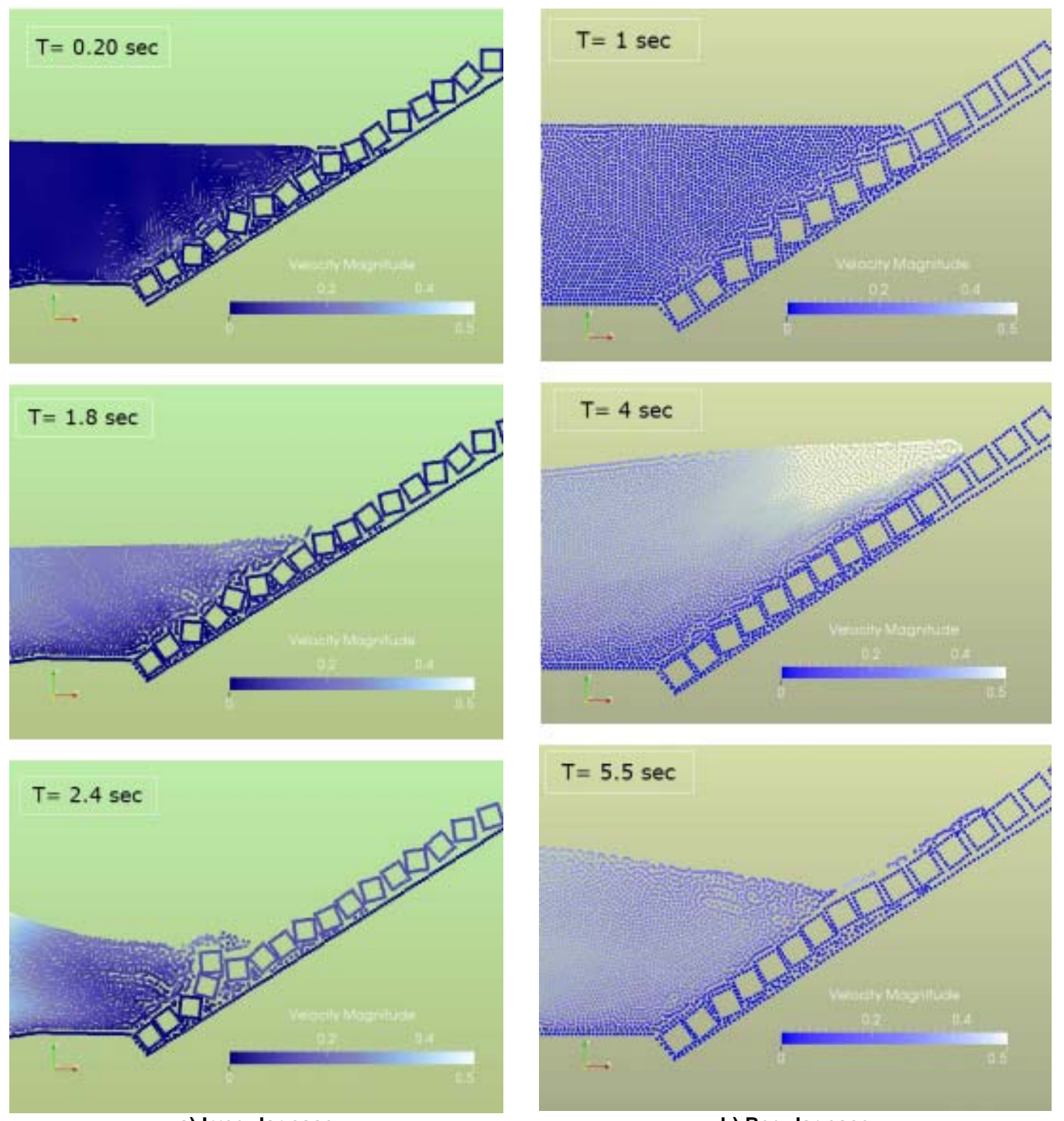

Figure 21. Displacement of armor units due to wave impact

b) Regular case

In the regular case, armor units recede from the contact surface with breakwater body in wave run down due to fluid force effect. This phenomenon (named "Clamping"), which is not seen in rock and/or concrete armor layers with complex geometries, happens in one layer concrete cubic armor layer, due to more contact surface of units with each other. This phenomenon is also reported in Van Buchem (Van Buchem, 2009). In his experiments, only one armor unit had been excluded for stability parameter of 5 .

Comparing the stability of armor layer in both regular and irregular cases, it is more possible that a unit moves toward instability in the irregular case. Also, in regular placement, destructive effect of wave force in run down, reduces due to group displacement of armor units.

\section{CONCLUSIONS}

Boundary treatments like Repulsive Forces and Lubrication Forces are basically developed to simulate contact of miso scale suspended solid particles. In this study, the capability of implemented 
Repulsive Force boundary condition algorithm in simulating macro behavior of solid bodies is evaluated. In the present study, performance of two types of rigid boundary condition (repulsive force and dynamic) is investigated. Dynamic boundary condition, despite the satisfactory results in the modeling of fluid and rigid body interaction, shows significant error in the problems with the purpose of rigid body interaction modeling. Results of this study revealed that, it is possible to model the hydrodynamic phenomena and fluid-rigid boundary interaction with suitable accuracy by the promotion of the dynamic boundary condition and its combination with ghost particle's boundary condition. However, this type of boundary condition does not have good performance in the modeling of interactions between rigid bodies.

On the other hand, repulsive boundary condition despite variations in the pressure estimation near rigid boundaries, is able to model interaction between fluid and rigid body motion and also, the contact between other rigid boundaries (in the fluid media) at an acceptable level. However, in the collision modeling, both boundary conditions do not have good and satisfactory performance. Therefore, in order to model hydrodynamic phenomena, which include body collision and of course, momentum transform, it is required to develop a boundary condition with the property of momentum and kinetic energy conservations.

A non-cohesive contact model has been developed and validated against other common boundary conditions. Using this approach, contact of arbitrary shapes (square, circle, wedge, etc.) can be modeled using the same contact detection algorithm and the same contact model. Also, non-spherical bodies can be modeled easily. Using the developed contact model, effect of armor layout on displacement of cubic concrete armors has been investigated against Van Buchem experiments and concluded that in the irregular case it is more possible that a unit moves toward instability.

\section{REFERENCES}

Adams, G., \& Nosonovsky, M. (2000). Contact modeling-forces. Tribology International, 33(5), 431-442.

Gomez-Gesteira, M., Rogers, B. D., Crespo, A. J., Dalrymple, R. A., Narayanaswamy, M., \& Dominguez, J. M. (2012). SPHysics-development of a free-surface fluid solver-Part 1: Theory and formulations. Computers \& Geosciences, 48, 289-299.

Hashemi, M., Fatehi, R., \& Manzari, M. (2012). A modified SPH method for simulating motion of rigid bodies in Newtonian fluid flows. International Journal of Non-Linear Mechanics, 47(6), 626-638.

Hertz, H. (1881). On the contact of elastic solids. J. reine angew. Math, 92(156-171), 110.

Ladd, A., \& Verberg, R. (2001). Lattice-Boltzmann simulations of particle-fluid suspensions. Journal of Statistical Physics, 104(5-6), 1191-1251.

Liu, M., Shao, J., Li, J., \& Fu, S. (2011). A New Solid Boundary Treatment Algorithm For Smoothed Particle Hydrodynamics. Paper presented at the AIP Conference Proceedings.

Monaghan, J., \& Kos, A. (1999). Solitary waves on a Cretan beach. Journal of waterway, port, coastal, and ocean engineering, 125(3), 145-155.

Monaghan, J. J. (1994). Simulating free surface flows with SPH. Journal of computational physics, 110(2), 399-406.

Pazouki, A., Serban, R., \& Negrut, D. (2014). A Lagrangian-Lagrangian Framework for the Simulation of Rigid and Deformable Bodies in Fluid Multibody Dynamics (pp. 33-52): Springer.

Peskin, C. S. (1977). Numerical analysis of blood flow in the heart. Journal of computational physics, 25(3), 220-252.

Rogers, B. D., Dalrymple, R. A., \& Stansby, P. K. (2009). SPH modeling of floating bodies in the surf zone. 01613782, 204-215.

Van Buchem, R. (2009). Stability of a single top layer of cubes. MSc-Thesis, Delft University of Technology, Delft. 